\title{
On Maximal, Discrete, and Area Operators
}

\author{
Chunping Xie \\ Department of Mathematics, Milwaukee School of Engineering
}

Received: November 08, 2014 / Accepted: December 03, 2014 / Published: January 25, 2015.

Abstract: In this paper, we study the boundednesses of maximal operator $g_{*}$, the discrete operator $g_{d}$, and the area operator $A$ on Bergman spaces.

Key words: Area operator, Bergman Space, Discrete Maximal operator, Hardy Space, maximal operator

\section{Introduction}

Let $\mathbb{D}=\{z:|z|<1\}$ denote the unit disk in the complex plane and $\mathbb{T}=\partial \mathbb{D}=\{z:|z|=1\} \quad$ its boundary, the unit circle. Littlewood-Paley operators have been well known, studied by a lot of people, and used to characterize function spaces, such as Hardy spaces, etc. In this paper, we mainly consider the characterizations of classical Bergman spaces by these operators. Here are two Littlewood-Paley operators we have studied:

$$
\begin{gathered}
g_{*}(f)(z)=\left(\int_{0}^{1}(1-r) \sup _{\rho<r}\left|f^{\prime}(\rho z)\right|^{2} d r\right)^{1 / 2} \\
g_{d}(f)(z)=\left(\sum_{n=0}^{\infty} 2^{-2 n}\left|f^{\prime}\left(r_{n} z\right)\right|^{2} d r\right)^{1 / 2}
\end{gathered}
$$

where $r_{n}=1-2^{-n}$ and $z \in \mathbb{D} \cup \mathbb{T}$.

A Luzin area function is defined to be

$$
A(f)(z)=\int_{D_{z}}\left|f^{\prime}(w)\right|^{2} d m(w)
$$

where

$D_{z}=$ the convex hull of $\left\{|w|<\frac{1}{2}|z|\right\} \cup\{z\}$.

For $0<p<\infty, H^{p}(\mathbb{D})$ is the usual Hardy

Corresponding author: Chunping Xie, Department of Mathematics, Milwaukee School of Engineering. E-mail: xie@msoe.edu. space of functions analytic on the unit disk and the norm of $f$ in $H^{p}$ is as follows

$$
\|f\|_{H^{p}}=\sup _{0 \leq r<1}\left(\int_{0}^{2 \pi} \mid f\left(\left.r e^{i \theta}\right|^{p} \frac{d \theta}{2 \pi}\right)^{1 / p}<\infty .\right.
$$

It is standard that for $f \in H^{p}$, its nontangential limit exists almost everywhere on $\mathbb{T}$,

$$
\lim _{r \rightarrow 1^{-}} f\left(r e^{i \theta}\right)=f\left(e^{i \theta}\right)
$$

and

$$
\|f\|_{H^{p}}=\left\|f\left(e^{i \theta}\right)\right\|_{L^{P}(\mathbb{T})} .
$$

we refer readers to [11] for Hardy space theory.

For $0<p<\infty$, the Bergman space $A^{p}(\mathbb{D})$ is defined as the set of all functions $f$ analytic in $\mathbb{D}$ with the following norm

$$
\|f\|_{\mathbb{A}^{p}(\mathbb{D})}=\left(\int_{D}|f(z)|^{p} d m(z)\right)^{1 / p}<\infty
$$

where $z=x+i y$ and $d m(z)=\frac{d x d y}{\pi}$ in $\mathbb{D}$, the normalized area measure on $\mathbb{D}$. It is well known that $\mathrm{A}^{p}(\mathbb{D})$ is a Bnach space when $1 \leq p<\infty$ and when $0<p<1$, the space $A^{p}(\mathbb{D})$ is a quasi-Banach space with $p$-norm $\|f\|_{A^{p}(\mathbb{D})}^{p}$.

The main results of this note are to prove that for $f$ is analytic, $f$ in the Bergman space $\|f\|_{A^{p}(\mathbb{D})}$ if and only if $g_{*}(f)$ or $g_{d}(f)$ is in $L^{p}(\mathbb{D})$ and the similar results also obtained for the area operator A. 
Throughout this paper, for two nonnegative expressions $P$ and $Q, P \lesssim Q$ means that there exists a positive constant $C$, not depending on the main factors, such that $P \leq C Q$, and $P \approx Q$ means that $P \lesssim Q$ and $Q \lesssim P$.

\section{Main Results and Their Proofs}

For our convenience and reference, all main results and useful results are stated in this section and proofs are also given with some remarks.

When we prove Theorem 4 and 5 we need the following results on Hardy spaces due to Miroslav Pavlović, see Theorem 1 in [10].

Lemma 1. Let $0<p<\infty$. For an analytic function, the following conditions are mutually equivalent:

(a) $f \in H^{p}$;

(b) $g_{*}(f) \in L^{p}(\mathbb{T})$;

(c) $g_{d}(f) \in L^{p}(\mathbb{T})$.

Furthermore, there are constants $C_{1}, C_{2}, C_{3}$ independent of $f$ such that

$$
\begin{aligned}
& \|f\|_{H^{p}} \leq C_{1}\left\|g_{*}(f)\right\|_{L^{p}(\mathbb{D})} \leq \\
& C_{2}\left\|g_{d}(f)\right\|_{L^{p}(\mathbb{D})} \leq C_{3}\|f\|_{H^{p}} .
\end{aligned}
$$

In order to prove the second inequality of Theorem 4 , we need a Theorem in [11] with $\alpha=0$ (Theorem 2.30 on page 69$)$. We state it as a lemma here.

Lemma 2. Suppose $0<p \leq 1$. Then there exists a sequence $\left\{a_{k}\right\}$ in $\mathbb{D}$ such that $\mathrm{A}^{p}(\mathbb{D})$ consists of functions of the form

$$
f(z)=\sum_{k=1}^{\infty} c_{k} \frac{1-\left|a_{k}\right|^{2}}{\left(1-\bar{a}_{k} z\right)^{\frac{2}{p}+1}}, \quad z \in \mathbb{D}
$$

where $\left\{c_{k}\right\}$ belongs to the sequence space $l^{p}$ and the series converges in the norm topology of $\mathrm{A}^{p}(\mathbb{D})$.

Moreover,

$$
\|f\|_{A^{p}(D)}^{p} \leq \sum_{k=1}^{\infty}\left|c_{k}\right|^{p}\left\|f_{k}\right\|_{\mathrm{A}^{p}(\mathbb{D})}^{p}
$$

where

$$
f_{k}(z)=\frac{1-\left|a_{k}\right|^{2}}{\left(1-\bar{a}_{k} z\right)^{\frac{2}{p}+1}}
$$

and there is a constant $C$ such that

$$
C^{-1}\left(\sum_{k=1}^{\infty} c_{k}\right)^{1 / p} \leq\|f\|_{A^{p}(\mathbb{D})} \leq C\left(\sum_{k=1}^{\infty} c_{k}\right)^{1 / p} .
$$

A relationship between Littlewood-Paley operators is listed here and we will need it in the proof of Theorem 5.

Lemma 3. For any $f \in A^{p}(\mathbb{D})$, $g_{d}(f) \lesssim g_{*}(f)$.

$$
\begin{aligned}
g_{*}(f)^{2}(z) & =\int_{0}^{1}(1-s) \sup _{\rho<s}\left|f^{\prime}(\rho z)\right|^{2} d s \\
& =\sum_{n=0}^{\infty} \int_{r_{n}}^{r_{n+1}}(1-s) \sup _{\rho<s}\left|f^{\prime}(\rho z)\right|^{2} d s \\
& \geq \sum_{n=0}^{\infty} \sup _{\rho<r_{n}}\left|f^{\prime}(\rho z)\right|^{2} \int_{r_{n}}^{r_{n+1}}(1-s) d s \\
& \geq \frac{3}{8} g_{d}(f)^{2}(z) .
\end{aligned}
$$

Theorem 4. For $0<p<\infty$ and $f \in A^{p}(\mathbb{D})$ with $f(0)=0$, the following are true

$$
C_{p}^{\prime}\|f\|_{A^{p}(\mathbb{D})} \leq\left\|g_{*}(f)\right\|_{L^{p}(\mathbb{D})} \leq C_{p}^{\prime \prime}\|f\|_{A^{p}(\mathbb{D})}
$$

where $C_{p}^{\prime}$ and $C_{p}^{\prime \prime}$ are constants depending only on $p$.

$$
\begin{aligned}
& \text { Moreover } \\
& \|f\|_{A^{p}(\mathbb{D})} \approx|f(0)|+\left\|g_{*}(f)\right\|_{L^{p}(\mathbb{D})}, \quad 1 \leq p<\infty
\end{aligned}
$$

and

$$
\|f\|_{A^{p}(\mathbb{D})}^{p} \approx|f(0)|^{p}+\left\|g_{*}(f)\right\|_{L^{p}(\mathbb{D})}^{p}, \quad 0<p<1
$$

The basic idea of the proof of this theorem is due to Chen and Ouyang in [5] and based on some original results by Littlewood-Paley, Zygmund, and Lemma 1.

Proof of Theorem 4 For $0<p<\infty$, let $f_{r}(z)=f(r z) \quad$ for $\quad 0<r<1 \quad$ and $\quad z \in \mathbb{D}$, $z=r e^{i \theta}, 0 \leq \theta \leq 2 \pi$, we have 
28

On maximal, Discrete, and Area Operators

$$
\begin{aligned}
\int_{D}|f(z)|^{p} d m(z) & =\int_{0}^{2 \pi} \int_{0}^{1} r\left|f\left(r e^{i \theta}\right)\right|^{p} \frac{d r d \theta}{\pi} \\
& =2 \int_{0}^{1} r \int_{0}^{2 \pi}\left|f_{r}\left(e^{i \theta}\right)\right|^{p} \frac{d r d \theta}{2 \pi}
\end{aligned}
$$

By (1), it follows that

$$
\begin{aligned}
& \int_{D}|f(z)|^{p} d m(z) \\
& \approx 2 \int_{0}^{1} r \int_{0}^{2 \pi}\left|g_{*}\left(f_{r}\right)\left(e^{i \theta}\right)\right|^{p} \frac{d r d \theta}{2 \pi} \\
& \approx \int_{0}^{1}\left\|g_{*}\left(f_{r}\right)\right\|^{p} r d r \\
& =\int_{0}^{1} \int_{0}^{2 \pi}\left[\int_{0}^{1}(1-s) \sup _{\rho<s}\left|f_{r}\left(\rho e^{i \theta}\right)\right|^{2} d s\right]^{p / 2} \frac{d \theta}{2 \pi} r d r \\
& =\int_{0}^{1} \int_{0}^{2 \pi}\left[\int_{0}^{1}(1-s) \sup _{\rho<s}\left|f^{\prime}\left(r \rho e^{i \theta}\right)\right|^{2} r^{2} d s\right]^{p / 2} \frac{d \theta}{2 \pi} r d r \\
& \lesssim\left\|g_{*}(f)\right\|_{L^{p}(\mathbb{D})}^{p} .
\end{aligned}
$$

The first inequality of Theorem 4 is proved.

For $0<p \leq 1$, by Lemma 2, we get

$$
f(z)=\sum_{k=1}^{\infty} c_{k} f_{k}(z)
$$

Standard calculations give us

$$
\begin{aligned}
& \left|f_{k}(\rho z)\right|^{2}= \\
& \left(1-\left|a_{k}\right|^{2}\right)\left(\frac{2}{p}+1\right)^{2}\left|\bar{a}_{k}\right|^{2} \frac{1}{\left|1-\bar{a}_{k} \rho z\right|^{2\left(\frac{2}{p}+2\right)}}
\end{aligned}
$$

At this point we are in need of a very useful fact which is easy to check. For $0<t \leq 1, z \in \mathbb{D}$, we have

$$
|1-t z| \leq(1-t)+|1-z| \leq 3|1-t z| .
$$

Now we have

$$
\begin{aligned}
& g_{*}\left(f_{k}\right)(z) \\
\lesssim & \left(1-\left|a_{k}\right|^{2}\right)\left(\int_{0}^{1} \sup _{\rho<r} \frac{1-r}{\left[(1-\rho)+\left|1-\bar{a}_{k} z\right|\right]^{2\left(\frac{2}{p}+2\right)}} d r\right)^{1 / 2}
\end{aligned}
$$

$$
\begin{aligned}
& \lesssim\left(1-\left|a_{k}\right|^{2}\right)\left(\int_{0}^{1} \sup _{\rho<r} \frac{1}{\left[(1-\rho)+\left|1-\bar{a}_{k} z\right|\right]^{2\left(\frac{2}{p}+1\right)+1}} d r\right)^{1 / 2} \\
& =\left(1-\left|a_{k}\right|^{2}\right)\left(\int_{0}^{1} \frac{1}{\left[(1-r)+\left|1-\bar{a}_{k} z\right|\right]^{2\left(\frac{2}{p}+1\right)+1}} d r\right)^{1 / 2} \\
& \lesssim\left(1-\left|a_{k}\right|^{2}\right) \frac{1}{\left|1-\bar{a}_{k} z\right|^{\frac{2}{p}+1}} .
\end{aligned}
$$

Therefore, for $f=\sum_{k} c_{k} f_{k} \quad$ with $\sum_{k}\left|c_{k}\right|^{p}<\infty$, we have

$$
\begin{aligned}
& \int_{D}\left|g_{*}(f)(z)\right|^{p} d m(z) \\
& \leq \sum_{k=1}^{\infty}\left|c_{k}\right|^{p} \int_{D}\left|g_{*}\left(f_{k}\right)(z)\right|^{p} d m(z) \\
& \lesssim \sum_{k=1}^{\infty}\left|c_{k}\right|^{p}\left(1-\left|a_{k}\right|^{2}\right)^{p} \int_{D} \frac{1}{\left|1-\bar{a}_{k} z\right|^{2+p}} d m(z) \\
& \lesssim \sum_{k=1}^{\infty}\left|c_{k}\right|^{p} .
\end{aligned}
$$

Note that

$$
\int_{D} \frac{1}{\left|1-\bar{a}_{k} z\right|^{2+p}} d m(z) \approx \frac{1}{\left(1-\left|a_{k}\right|^{2}\right)^{p}} .
$$

Next for the case of $1<p<\infty$, if $f \in \mathrm{A}^{p}(\mathbb{D})$, then $f$ has the integral representation [11] (Theorem 2.2 on page 40),

$$
f(z)=\int_{D} \frac{f(w)}{(1-\bar{w} z)^{2}} d m(w), \quad \forall z \in \mathbb{D} .
$$

So

$\left|f^{\prime}(\rho z)\right| \leq(1-|\rho z|)^{-1 / 2} \int_{D} \frac{|f(w)|}{|1-\rho \bar{w} z|^{5 / 2}} d m(w)$.

Hence we have

$g_{*}(f)^{2}(z) \lesssim$

$\int_{0}^{1} \sup _{\rho<r} \frac{1-r}{(1-|\rho z|)}\left[\int_{D} \frac{|f(w)|}{|1-\rho \bar{w} z|^{5 / 2}} d m(w)\right]^{2} d r$. 
By (1), we have the following estimate

$$
3|1-\rho \bar{w} z| \geq\left(1-\frac{\rho}{r}\right)+|1-r \bar{w} z| .
$$

Therefore, we obtain

$$
g_{*}(f)^{2}(z) \lesssim \int_{0}^{1}\left[\int_{D} \frac{|f(w)|}{|1-r \bar{w} z|^{5 / 2}} d m(w)\right]^{2} d r
$$

By Minkowski's integral inequality, we have

$$
\begin{aligned}
& g_{*}(f)^{2}(z) \\
& \lesssim\left[\int_{D}|f(w)|\left(\int_{0}^{1} \frac{1}{|1-r \bar{w} z|^{5}}\right)^{1 / 2} d m(w)\right]^{2} \\
& \lesssim\left[\int_{D}|f(w)|\left(\int_{0}^{1} \frac{1}{[(1-r)+|1-\bar{w} z|]^{5}}\right)^{1 / 2} d m(w)\right]^{2} \\
& \lesssim\left[\int_{D} \frac{|f(w)|}{|1-\bar{w} z|^{2}} d m(w)\right]^{2} .
\end{aligned}
$$

But,

$$
f \mapsto \int_{D} \frac{|f(w)|}{|1-\bar{w} z|^{2}} d m(w)
$$

is bounded on $L^{p}(\mathbb{D})$ for $1<p<\infty$. The proof of Theorem 4 is completed.

The next theorem is regarding the characterization of analytic function $f$ in Bergman space $A^{p}(\mathbb{D})$ by the discrete Littlewood-Paley operator $g_{d}(f)$.

Theorem 5. For $0<p<\infty$ and $f \in \mathrm{A}^{p}(\mathbb{D})$ with $f(0)=0$, there exist constants $C_{p}^{\prime}$ and $C_{p}^{\prime \prime}$ depending only on $p$ such that

$$
C_{p}^{\prime}\|f\|_{A^{p}(\mathbb{D})} \leq\left\|g_{d}(f)\right\|_{L^{p}(\mathbb{D})} \leq C_{p}^{\prime \prime}\|f\|_{A^{p}(\mathbb{D})} .
$$

Moreover

$$
\|f\|_{A^{p}(\mathbb{D})} \approx|f(0)|+\left\|g_{d}(f)\right\|_{L^{p}(\mathbb{D})}, \quad 1 \leq p<\infty
$$

and

$$
\|f\|_{A^{p}(\mathbb{D})}^{p} \approx|f(0)|^{p}+\left\|g_{d}(f)\right\|_{L^{p}(\mathbb{D})}^{p}, \quad 0<p<1
$$

The proof of the first half of the inequality of Theorem 5 is similar to that of Theorem 4 but we have to be careful with the change from continuous case to discrete case.

Proof of Theorem 5 For $0<p<\infty$ and $f_{r}(z)=f(r z) \quad 0<r<1$ and $z \in \mathbb{D}, \quad z=r e^{i \theta}$, by (1) and standard calculations, we have

$$
\begin{aligned}
\int_{D}|f(z)|^{p} d m(z) & =\int_{0}^{2 \pi} \int_{0}^{1} r\left|f\left(r e^{i \theta}\right)\right|^{p} \frac{d r d \theta}{\pi} \\
& =2 \int_{0}^{1} r \int_{0}^{2 \pi}\left|f_{r}\left(e^{i \theta}\right)\right|^{p} \frac{d r d \theta}{2 \pi} \\
& \approx \int_{0}^{1}\left\|g_{d}\left(f_{r}\right)\right\|^{p} r d r \\
& \lesssim\left\|g_{d}(f)\right\|_{L^{p}(\mathbb{D})}^{p} .
\end{aligned}
$$

The last inequality holds because

$g_{d}\left(f_{r}\right)\left(e^{i \theta}\right) \leq\left(\sum_{n=0}^{\infty} 2^{-2 n} \mid f^{\prime}\left(\left.r r_{n} e^{i \theta}\right|^{2}\right)^{1 / 2}=g_{d}(f)(z)\right.$

The second half of Theorem 5 follows immediately from Theorem 4 and Lemma 3. This completes the proof of Theorem 5.

In the next part, we are going to consider the classical area integral operator, also called the Luzin area operator on Bergman space. To state our results we need the following due to Calderó n. In fact it is a special case of Calder ó n's theorem. See Theorem 1.3 in [10].

Lemma 6. Let $0<p<\infty$. Then $f \in H^{p}(\mathbb{D})$ if and only if $A(f) \in L^{p}(\mathbb{D})$.

Theorem 7. For $0<p<\infty, f \in A^{p}(\mathbb{D})$ with $f(0)=0$, there exists a constant $C$ such that

$$
\|f\|_{A^{p}(\mathbb{D})} \leq C\|A(f)\|_{L^{p}(\mathbb{D})} .
$$

Proof For $0<p<\infty$, let $f_{r}(z)=f(r z)$, $0<r<1$. By Lemma 6 , we have 


$$
\begin{aligned}
& \int_{D}|f(z)|^{p} d m(z) \\
& =2 \int_{0}^{1}\left[\int_{0}^{2 \pi}\left|f\left(r e^{i \theta}\right)\right|^{p} \frac{d \theta}{2 \pi}\right] r d r \\
& \approx 2 \int_{0}^{1}\left[\int_{0}^{2 \pi}\left|A\left(f_{r}\right)\left(e^{i \theta}\right)\right|^{p} \frac{d \theta}{2 \pi}\right] r d r \\
& \leq C\|A(f)\|_{L^{p}(\mathbb{D})} .
\end{aligned}
$$

Here in the last step we used the fact that

$$
A\left(f_{r}\right)(z) \leq A(f)(z) .
$$

The converse of Theorem 7 is not as good as those of Theorem 4 and 5 when $1 \leq p<\infty$.

Theorem 8. For $0<p<1, f \in \mathrm{A}^{p}(\mathbb{D})$ with $f(0)=0$, there exists a constant $C$ such that

$$
\|A(f)\|_{A^{p}(\mathbb{D})} \leq C\|f\|_{L^{p}(\mathbb{D})} .
$$

By Lemma 2, for $0<p<1$,

$$
f(z)=\sum_{k=1}^{\infty} c_{k} f_{k}(z)
$$

where

$$
f_{k}(z)=\frac{1-\left|a_{k}\right|^{2}}{\left(1-\bar{a}_{k} z\right)^{\frac{2}{p}+1}} .
$$

So we have

$$
\begin{aligned}
& A\left(f_{k}\right)(z) \\
& \lesssim\left(1-\left|a_{k}\right|^{2}\right)\left[\int_{D_{z}} \frac{1}{\left|1-\bar{a}_{k} w\right|^{2\left(\frac{2}{p}+2\right)}} d m(w)\right]^{1 / 2}
\end{aligned}
$$

and

$$
\begin{aligned}
& \int_{D}|A(f)(z)|^{p} d m(z) \leq \\
& \sum_{k=1}^{\infty}\left|c_{k}\right|^{p}\left(1-\left|a_{k}\right|^{2}\right)^{p}\left[\int_{D_{z}} \frac{1}{\left|1-\bar{a}_{k} w\right|^{2\left(\frac{2}{p}+2\right)}} d m(w)\right]^{p / 2} .
\end{aligned}
$$

To get through our proof, we need to estimate the inner integral in the last line by variable changes.

$$
\begin{aligned}
& \int_{D_{z}} \frac{1}{\left|1-\bar{a}_{k} w\right|^{2\left(\frac{2}{p}+2\right)}} d m(w) \\
& \leq \int_{|w| \leq|z|} \frac{1}{\left|1-\bar{a}_{k} w\right|^{2\left(\frac{2}{p}+2\right)}} d m(w) \\
& =\int_{D} \frac{1}{\left|1-\bar{a}_{k}\right| z|w|^{2\left(\frac{2}{p}+2\right)}} \frac{1}{|z|^{2}} d m(w) \\
& \approx \frac{1}{|z|^{2}} \frac{1}{\left(1-\left|a_{k} z\right|^{2}\right)^{2\left(\frac{2}{p}+1\right)}} .
\end{aligned}
$$

Therefore we have

$$
\begin{aligned}
& \int_{D}|A(f)(z)|^{p} d m(z) \\
& \leq \int_{D} \frac{1}{|z|^{p}} \frac{1}{\left(1-\left|a_{k} z\right|^{2}\right)^{2+p}} d m(z) \\
& \leq \frac{1}{\left(1-\left|a_{k}\right|^{2}\right)^{2}} \int_{D} \frac{1}{|z|^{p}} \frac{1}{\left(1-\left|a_{k} z\right|^{2}\right)^{p}} d m(z)
\end{aligned}
$$$$
\leq \frac{1}{\left(1-\left|a_{k}\right|^{2}\right)^{2}} \int_{D} \frac{1}{|z|^{p}} \frac{1}{\left(1-|z|^{2}\right)^{p}} d m(z)
$$$$
\lesssim \frac{1}{\left(1-\left|a_{k}\right|^{2}\right)^{2}} \int_{0}^{2 \pi} \int_{0}^{1} r^{1-p}(1-r)^{-p} d r d \theta
$$

$$
\lesssim \frac{1}{\left(1-\left|a_{k}\right|^{2}\right)^{2}} B(2-p, 1-p)
$$

where $B(a, b)=\int_{0}^{1} t^{a-1}(1-t)^{b-1} d t \quad$ is the Beta function. Hence

$$
\int_{D}|A(f)(z)|^{p} d m(z) \lesssim \sum_{k}\left|c_{k}\right|^{p} .
$$

We are done with the proof of Theorem 8 .

\section{References}

[1] S. Axler Bergman Spaces and Their Operators, in Surveys of Some Recent Results in OPerator Theory(Volume 1, J. B. Conway and B. B. Morrel, Editors, Pitman Research Notes in Math 171 (1988), $1-50$.

[2] P. Ahern and J. Bruna Maximal and Area Integral Characterizations of Hardy-Sobolev Spaces in the Unit Ball of C ${ }^{\mathrm{n}}$, Rev. Mat. Iberoamericana 4 (1988), 123-153. 
[3] R. Aulaskari, J. Xiao, and R. Zhao On subspaces and subsets of BMOA and UBC, Analysis 15 (1995), 101-121.

[4] F. Chiarenza, M. Frasca, Morrey spaces and Hardy-Littlewood maximal function, Rend. Mat. Appl. 7 (1987), 273-279.

[5] Zeqian Chen and Wei Ouyang A Littlewood-Paley Type Theorem for Bergman Spaces, Acta Mathematica Scientia 33B(1) (2013), 150-154.

[6] Peter Duren Bergman Spaces, American Mathematical Society, SURV 100 (2004).

[7] Ashot E. Djrbashian and Faizo A. Shamoian Topics in the theory of $A_{\alpha}^{p}$ Spaces, Leipzig, Teubner (1988).

[8] J. B. Garnett, Bounded Analytic Functions, Academic Press, Orlando, Fla. (1981).

[9] J. Garcia Cuerva, J. Rubio de Francia, Weighted norm inequalities and related topics, North-Holland Math. Stud. (1985).

[10] Miroslav Pavlović On the Littlewood-Paley $g$-function and Calderon's Area Theorem, Expo. Math. 31 (2013), 169-195.

[11] Kehe Zhu Spaces of Holomorphic Functions in the Unit Ball, Springer, GTM, New York (2005). 